\title{
Spectral Dependence of Fluorescence Enhancement in LH2-Au Nanoparticle Hybrid Nanostructures
}

\author{
L. Bujak ${ }^{a}, *$ T.H.P. Brotosudarmo ${ }^{b}$, N. Czechowski ${ }^{a}$, M. Olejnik ${ }^{a}$, K. Ciszak $^{a}$, \\ R. Litvin ${ }^{a}$, R.J. COGDell ${ }^{b}$, W. Heiss ${ }^{c}$ AND S. MACKOWSKI ${ }^{a}$ \\ ${ }^{a}$ Institute of Physics, Nicolaus Copernicus University, Grudziądzka 5, 87-100 Toruń, Poland \\ ${ }^{b}$ Glasgow Biomedical Research Centre, University of Glasgow, United Kingdom \\ ${ }^{c}$ Institute for Semiconductor Physics, University of Linz, Austria
}

\begin{abstract}
We report on the influence of plasmon resonance in spherical gold nanoparticles on the optical properties of light-harvesting complex LH2 from the purple bacteria Rhodopseudomonas palustris. Systematic studies as a function of the excitation energy and the separation distance indicate that metal enhanced fluorescence shows strong dependence upon both of these parameters. We observe substantial increase of the fluorescence from LH2 complex in a hybrid nanostructure with $12 \mathrm{~nm}$ silica spacer. On the other hand, the enhancement measured with laser tuned into the plasmon resonance is almost threefold compared to the off-resonance configuration. The enhancement of fluorescence intensity originates in both cases from the increase of carotenoid absorption in the LH2 complex.
\end{abstract}

PACS: 87.80.Dj, 78.47.D-, 78.67.Qa, 82.35.Np

\section{Introduction}

Plasmons, free electron oscillations in metallic nanometric materials, enable strong modifications of the electromagnetic field at the nanoscale [1] thus becoming scientific inspiration for many research fields including photovoltaics and biosensors $[2,3]$. One of the most spectacular effects associated with plasmon excitations in metallic nanoparticles is metal-enhanced fluorescence [4], i.e. the increase of the radiative rate of a fluorophore due to plasmon coupling. This effect depends among others upon the separation between a fluorophore and metallic nanoparticle as well as their spectral properties [5]. Namely the largest values of fluorescence enhancement have been observed for distances around 10$20 \mathrm{~nm}$ and for metallic nanoparticles with plasmon resonances matched spectrally to the emission range of the fluorophore [5]. Analogous consideration is also valid for plasmon-induced increase of absorption rate [6-8], as observed for photosynthetic complexes responsible for absorption of light. When the separation gets shorter, the nonradiative energy transfer from the fluorophore to the metallic nanoparticle takes place leading to efficient quenching of fluorescence.

In this work we study the fluorescence properties of a hybrid nanostructure comprising spherical Au nanoparticles and light-harvesting complex LH2 from purple bacteria excited on- and off-resonance with respect to the plasmon resonance. The distance between them is controlled via $\mathrm{SiO}_{2}$ spacer, whose thickness varies from 4 to $40 \mathrm{~nm}$. For both excitation wavelengths we observe fluorescence enhancement for the spacer thickness of $12 \mathrm{~nm}$, but the on-resonance case $(485 \mathrm{~nm})$ yields three times stronger effect compared to the off-resonance (405 nm). The insensitivity of the fluorescence decay time on the spacer thickness for both excitation wavelengths points towards

\footnotetext{
* corresponding author; e-mail: bujak@fizyka.umk.pl
}

increase of the absorption rate in the light-harvesting complexes coupled to plasmon excitations in metallic nanoparticles.

\section{Materials and methods}

The LH2 complexes from Rps. palustris were prepared as described elsewhere [9]. The complexes were stored in Tris buffer with $0.1 \%$ LDAO. Spherical Au nanoparticles were synthesized by reducing chloroauric acid $\mathrm{HAuCl}_{4}$ with sodium citrate and dispersed in toluene. The average diameter of the gold nanoparticles was $5 \mathrm{~nm}$, which results in plasmon resonance maximum at $530 \mathrm{~nm}$. The hybrid nanostructure was prepared by first spin coating the Au nanoparticles on clean glass substrate. Next the samples were transferred to an e-beam evaporation chamber where silica layers were deposited. The thickness of the $\mathrm{SiO}_{2}$ layer was varied between 4 and $40 \mathrm{~nm}$. Finally, $10 \mu \mathrm{L}$ of the light-harvesting complexes dissolved in a polymer (PVA Sigma Aldrich) were spin-coated on top of $\mathrm{SiO}_{2}$ layer.

Absorption spectra of solutions of the LH2 complexes and $\mathrm{Au}$ nanoparticles were obtained using spectrophotometer (Perkin Elmer Lambda 2). Fluorescence measurements of hybrid nanostructures were carried out in a standard optical setup in a backscattering geometry. The laser excitation beams $(\lambda=405 \mathrm{~nm}$ and $\lambda=485 \mathrm{~nm})$ were focused using a lens with focal length of $30 \mathrm{~mm}$. Lasers can be operated in either continuous-wave or pulsed mode generating $30 \mathrm{ps}$ pulses with $80 \mathrm{MHz}$ repetition rate. Excitation power was controlled using notch filters to obtain $200 \mu \mathrm{W}$ on the sample surface. The emission was guided through a $150 \mu \mathrm{m}$ pinhole and focused on a slit of a 0.5 monochromator (Shamrock 500, Andor) coupled with a charge coupled device detector (iDus 420BV, Andor). In order to extract fluorescence of LH2 complexes we used a combination of a longpass filter (Chroma HQ850LP) and a bandpass filter (Chroma D880/40 m). Fluorescence decays were measured with a time-correlated single photon counting technique em- 
ploying a Becker \& Hickl SPC-150 card coupled with a fast avalanche photodiode detector (idQuantique id100-50). The overall temporal resolution of the experimental setup was about 100 ps. For each substrate and each excitation wavelength a series of ten measurements were taken on ten different locations across the sample.

\section{Results and discussion}

The pigment structure of the LH2 complex is shown in Fig. 1a. It contains 27 bacteriochlorophyll (BChl) molecules arranged in two rings: a strongly coupled ring of $18 \mathrm{BChl}$ (red) and a weakly interacting ring of $9 \mathrm{BChl}$ (green). The distances between the BChl molecules in the rings are 9 and $21 \AA$, respectively. The LH 2 complex includes also 9 carotenoid molecules (yellow) with all the pigments enclosed by a hydrophobic protein (not shown).
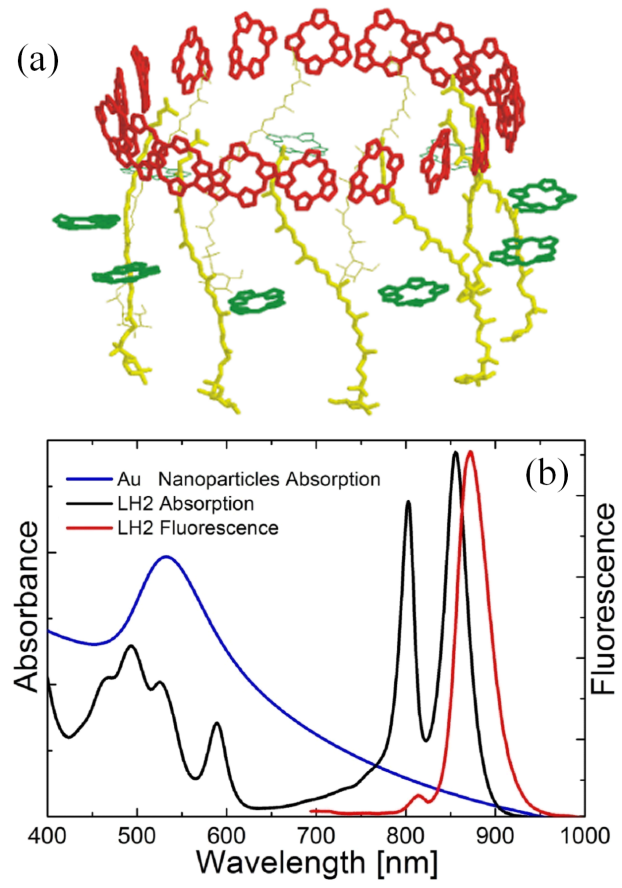

Fig. 1. (a) Pigment organization in the LH2 complex: nine weakly coupled bacteriochlorophyll molecules in B800 ring (green line), strongly coupled eighteen bacteriochlorophyll molecules in B850 ring (red line), and nine carotenoids (yellow line). (b) Optical absorption of Au nanoparticles in solution (black) compared with the absorption of the LH2 (blue). Emission spectrum of the LH2 complex is shown in red.

The optical spectra of gold nanoparticles and LH2 complexes are compared in Fig. 1b. Plasmon resonance in metallic nanoparticles appears at $530 \mathrm{~nm}$ (blue) and it overlaps with part of the LH2 absorption (black) associated mainly with carotenoids. The BChl molecules absorb predominantly at $800 \mathrm{~nm}$ (B800 ring) and $850 \mathrm{~nm}$ (B850 ring). The latter is also responsible for the fluorescence of LH2 (red in Fig. 1b), which due to strong coupling between BChl molecules has an excitonic character. The emission of the LH2 complex overlaps very slightly with the plasmon resonance of the gold nanoparticles. From the examination of the optical spectra of the components of the hybrid nanostructure we may expect that the dominant effect of plasmon excitations should be enhancement of the absorption of the light-harvesting complex.

The experimental procedure to evaluate the effect of plasmon excitations upon the optical properties of the LH2 complexes was based on measuring fluorescence spectra and fluorescence lifetimes for ten different spots across the sample for both excitation wavelengths of $405 \mathrm{~nm}$ and $485 \mathrm{~nm}$. In this way we account for possible inhomogeneity due to preparation of the hybrid nanostructures. Spin-coating - although producing relatively uniform layers - may also lead to local variations of the LH2 concentrations. At the same time, the thickness of the $\mathrm{SiO}_{2}$ layer could also show some degree of inhomogeneity which is typically below $2 \mathrm{~nm}$. The fluorescence spectra have been analyzed previously [10]: it has been shown that the shape of the emission is independent of the thickness of the $\mathrm{SiO}_{2}$ spacer, thus the strength of plasmon interaction. This indicates that the strength of enhancement shows no variation over the spectral range of the fluorescence emission, that is around $870 \mathrm{~nm}$.

In Fig. 2 we show normalized fluorescence transients measured for LH2 complexes separated by 4 and $12 \mathrm{~nm}$ thick $\mathrm{SiO}_{2}$ spacers from a layer of $\mathrm{Au}$ nanoparticles, as well as for the LH2 complexes deposited directly on a glass substrate. The data for $405 \mathrm{~nm}$ excitation, which is located away from the plasmon resonance (Fig. 2a), is compared with the results measured for LH2 complexes excited with $485 \mathrm{~nm}$ (Fig. 2b), which is much closer to the plasmon resonance of the Au nanoparticle. It is important to note that both lasers excite the emission of the $\mathrm{B} 850$ ring quite efficiently due to the energy transfer between carotenoids and BChl molecules [11]. First of all, the fluorescence decay features essentially no dependence upon the excitation wavelength, the differences of fluorescence lifetimes are comparable to the experimental accuracy. We also find weak dependence of the fluorescence decay times on the thickness of the $\mathrm{SiO}_{2}$ spacer.

At the same time the intensity of fluorescence emission extracted by integrating the decay curves displays strong dependence upon the thickness of the $\mathrm{SiO}_{2}$ spacer. The results obtained for the two laser excitation wavelengths are shown in Fig. 3. For both cases the enhancement factor features non-monotonic behavior: when reducing the spacer thickness from $40 \mathrm{~nm}$, which we consider our reference, the fluorescence intensity starts to increase reaching maximum for the hybrid nanostructure with the spacer of $12 \mathrm{~nm}$. Upon further reduction of the spacer thickness the intensity drops down. The overall dependence of the enhancement factor on the thickness of the dielectric spacer is pretty similar to the one measured for single fluorescent molecules coupled to spherical metallic nanoparticles [5]. Importantly, the enhancement of the LH2 emission measured for the off-resonance $405 \mathrm{~nm}$ excitation is significantly reduced compared to 


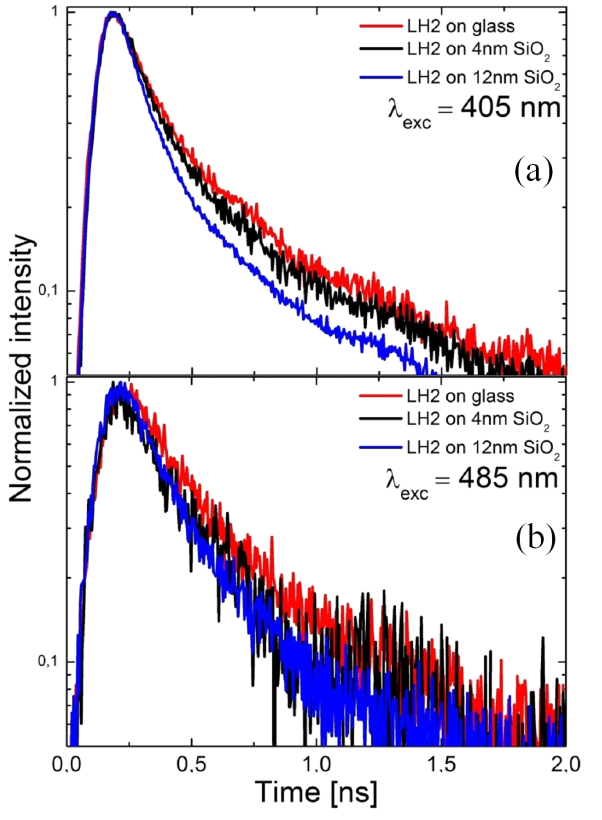

Fig. 2. Comparison of fluorescence decay curves measured for LH2 complexes deposited on glass and deposited on $\mathrm{Au}$ NPs with $4 \mathrm{~nm}$ and $12 \mathrm{~nm}$ thick spacer. The excitation wavelengths were (a) $405 \mathrm{~nm}$ and (b) $485 \mathrm{~nm}$.

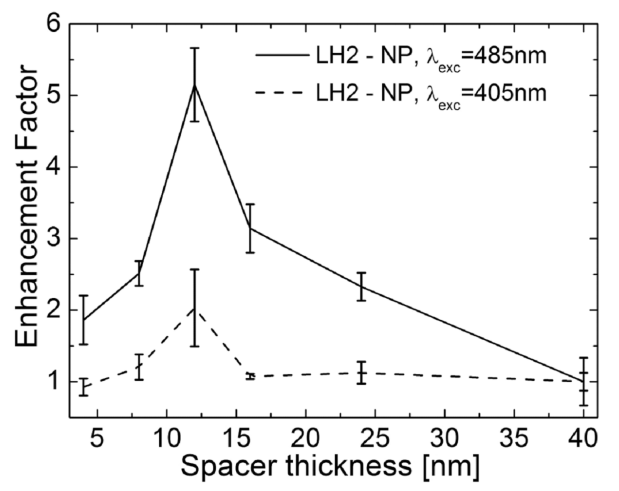

Fig. 3. Comparison of enhancement factor as a function of the spacer thickness for studied hybrid nanostructures. Solid line represents the on-resonance $485 \mathrm{~nm}$ excitation laser, while dashed line indicates the off-resonance $405 \mathrm{~nm}$ excitation laser.

the on-resonance excitation at $485 \mathrm{~nm}$. Indeed, the on-resonance excitation yields maximum enhancement of 12 for the $\mathrm{SiO}_{2}$ spacer with a thickness of $12 \mathrm{~nm}$, with an average value equal to 5 , as compared to the reference structure. In contrast, for the off-resonance $405 \mathrm{~nm}$ laser excitation the average enhancement factor for the $12 \mathrm{~nm}$ thick $\mathrm{SiO}_{2}$ spacer is only twofold. Nevertheless, the overall shape of the enhancement factor dependence on the spacer thickness is the same for both excitation wavelengths. Minute changes in fluorescence decays combined with significant increase of the emission intensity indicate that observed enhancement of fluorescence is due to enhancement in carotenoid absorption region of LH2 com- plex. Furthermore, the results, in particular the strong sensitivity of the fluorescence enhancement to the excitation wavelength points toward critical role of exciting plasmons in metallic nanoparticles for inducing spectral changes of the fluorophores.

\section{Conclusions}

Time resolved fluorescence spectroscopy of hybrid nanostructures composed of light-harvesting complex LH2 and gold nanoparticles reveal critical role of the wavelength of laser excitation on measured enhancement factor. On-resonance excitation induced strong, 5 -fold enhancement of the emission intensity, while off-resonance yield just a twofold enhancement. For both excitation wavelengths the qulitative dependence of the enhancement factor upon the thickness of a dieletric layer is pretty similar. The absence of plasmon induced changes on the fluorescence decay time in our hybrid nanostructures suggests the increase of absorption rate being the predominant mechanism responsible for observed increase of the fluorescence.

\section{Acknowledgments}

Financial support from the WELCOME program "Hybrid nanostructures as a stepping-stone towards efficient artificial photosynthesis" awarded by the Foundation for Polish Science is gratefully acknowledged. Work at University of Glasgow was supported by the Office of Basic Energy Sciences, Division of Materials Science and Engineering, U.S. Department of Energy under Award \#DESC0001035.

\section{References}

[1] S. Maier, Plasmonics: Fundamentals and Applications, Springer Science + Business Media LLC, New York 2007.

[2] H.A. Atwater, A. Polman, Nature Mater. 9, 205 (2010).

[3] J. Lee, P. Hernandez, J. Lee, A.O. Govorov, N.A. Kotov, Nature Mater. 6, 291 (2007).

[4] J.R. Lakowicz, Anal. Biochem. 298, 1 (2001).

[5] P. Bharadwaj, P. Anger, L. Novotny, Nanotechnology 18, 044017 (2007).

[6] S. Mackowski, J. Phys., Condens. Matter 22, 193102 (2010).

[7] N. Czechowski, P. Nyga, M.K. Schmidt, T.H.P. Brotosudarmo, H. Scheer, D. Piatkowski, S. Mackowski, Plasmonics 7, 115 (2012).

[8] I. Carmeli, I. Liberman, L. Kraverski, Z. Fan, A.O. Govorov, G. Markovich, S. Richter, Nano Lett. 10, 2069 (2010).

[9] T.H.P. Brotosudarmo, R. Kunz, P. Böhm, A.T. Gardiner, V. Moulisová, R.J. Cogdell, J. Köhler, Biophys. J. 97, 1491 (2009).

[10] Ł. Bujak, N. Czechowski, D. Piatkowski, R. Litvin, S. Mackowski, T.H.P. Brotosudarmo, R.J. Cogdell, S. Pichler, W. Heiss, Appl. Phys. Lett. 99, 173701 (2011).

[11] S. Wörmke, S. Mackowski, T.H.P. Brotosudarmo, A. Garcia, P. Braun, H. Scheer, E. Hofmann, C. Bräuchle, Appl. Phys. Lett. 90, 193901 (2007). 